\title{
Effect of Age and Body Mass Index on Surgical Treatment Outcomes in Patients with Carpal Tunnel Syndrome
}

\author{
Muzaffer GUNES ${ }^{1}$, Ersin OZEREN² \\ ${ }^{1}$ Aksaray University School of Medicine, Department of Neurology, Aksaray, Turkey \\ ${ }^{2}$ Aksaray University School of Medicine, Department of Neurosurgery, Aksaray, Turkey \\ Corresponding author: Muzaffer GUNES drmuzaffergunes@gmail.com
}

\section{ABSTRACT}

AIM: To investigate the effect of age, diabetes mellitus, and body mass index (BMI) on the outcomes of carpal tunnel syndrome (CTS) surgery in patients with moderate CTS according to neurophysiological classification.

MATERIAL and METHODS: Postsurgical outcomes were evaluated via a nerve conduction study (NCS) six to nine months after the surgery. Patients were divided into two groups according to neurophysiological classification based on the findings of postsurgical NCS: patients with mild CTS (partial recovery) or normal NCS (complete recovery) were classified as surgical success, and patients with moderate to more severe CTS were defined as surgical failure.

RESULTS: Forty-seven patients who underwent surgery for moderate CTS were included in the study. The mean age of the patients with surgical success $(47.3 \pm 10.9$ years $)$ was significantly lower than that of patients with surgical failure $(54.3 \pm 12.6$ years; $\mathrm{p}=0.048)$. The mean BMl of the patients with surgical success $\left(31.3 \pm 4.03 \mathrm{~kg} / \mathrm{m}^{2}\right)$ was significantly lower than that of patients with surgical failure $\left(36.75 \pm 5.5 \mathrm{~kg} / \mathrm{m}^{2} ; \mathrm{p}<0.001\right)$. Multivariate logistic regression analysis demonstrated that higher $\mathrm{BMI}(\mathrm{p}=0.003)$ and older age $(p=0.031)$ were independent prognostic factors for surgical failure. In addition, there was a statistically significant and strong correlation between postoperative CTS severity and BMI (rho=0.606, $p<0.001$ ).

CONCLUSION: Advanced age and high BMI negatively affect the success of surgical treatment in patients with CTS. Ensuring weight loss before surgery in CTS patients who have high BMI may increase the probability of treatment success.

KEYWORDS: Carpal tunnel syndrome, Nerve conduction studies, Surgical treatment outcomes, Body mass index, Diabetes mellitus

\section{INTRODUCTION}

$\longrightarrow$ arpal tunnel syndrome (CTS) is the most common mononeuropathy in humans. It occurs as a result of entrapment of the median nerve in the wrist (24). Age, female gender, obesity, and diabetes mellitus (DM) are important risk factors for CTS (3). Furthermore, this disease can have consequences such as disability and impaired quality of life (1). It is therefore important to select the most appropriate treatment, which includes surgical or nonsurgical methods. Mild and moderate CTS cases are treated using nonsurgical treatment methods (18). However, surgical treatment is used for patients who do not benefit from nonsurgical methods or in addition to nonsurgical methods in more severe cases
$(4,7,10)$. Therefore, surgical treatment is an important part of CTS treatment. However, some patients may not benefit at all from surgical treatment (5). The disease may progress in these people. For this reason, it is important to determine the underlying causes in patients who do not benefit from surgical treatment. This is because the number of patients who benefit from surgical treatment can be increased by using interventions that target these underlying causes. The present study is intended to investigate the effect of age, body mass index (BMI) and DM on the outcomes of CTS surgery in patients with moderate CTS according to their neurophysiological classification. 


\section{- MATERIAL and METHODS}

\section{Study Population}

$\mathrm{T}$ The current study was conducted retrospectively in patients who were operated at Aksaray University Training and Research Hospital between June 2015 and March 2019 with a diagnosis of moderate CTS according to their neurophysiological classification. From our database, we found that control electroneuromyography outcomes were available for only 47 of the 430 patients operated for moderate CTS, and the data from these patients were used for this study. We included patients older than 18 years. CTS surgery was performed after obtaining consent from all patients. We excluded patients who previously underwent surgery for CTS, pregnant women, and those with local infection in the wrist or systemic infection, cervical radiculopathy, brachial plexopathy, and generalized peripheral neuropathy.

The diagnosis of CTS was confirmed by the presence of clinical symptoms and an electrodiagnostic nerve conduction study (NCS) performed electrodiagnostically. The temperature of the hand was kept at greater than $32^{\circ} \mathrm{C}$ during the neurophysiological examination. The median and ulnar nerves were examined bilaterally during the NCS. Sensory transmissions of the median and ulnar nerves were obtained antidromically on the second and fifth fingers, respectively, using surface recording electrodes. Recording was performed using surface recording electrodes from the abductor pollicis brevis muscle during the median motor NCS and from the abductor digiti minimi muscle during the ulnar motor NCS. Motor and sensory conduction distal latencies (ms), amplitudes, and nerve conduction velocities $(\mathrm{m} / \mathrm{s})$ of the bilateral median and ulnar nerve were measured. Patients diagnosed with moderate CTS in at least one hand and who were operated for this reason were included in the study. A two-channel electroneuromyography device (Micromed SpAVia Giotto, 2-31021 Mogliano Veneto, Italy, 2014) was used for the electrodiagnostic diagnosis.

Patients who exhibited median neuropathy in the wrist with NCS were categorized into the mild, moderate, or severe CTS groups $(9,21)$. The mild CTS group consisted of patients with a sensory nerve action potential (SNAP) or mixed nerve action potential distal latency delay of $>3.2 \mathrm{~ms}$ and/or a SNAP amplitude below the lower limit of normal $(<16 \mu \mathrm{V})$. The moderate CTS group included patients with a median nerve motor distal latency prolongation of $>4.6 \mathrm{~ms}$ and who met the criteria for mild CTS. The severe CTS group consisted of patients whose median nerve compound muscle nerve action potential amplitude was below the lower limit of normal $(<5.0$ $\mathrm{mV}$ ) or patients without SNAP.

In our clinic, the height and weight of all patients is routinely measured and recorded before surgery. We retrieved the height and weight of the patients included in this study our database and calculated the patients' BMI $\left(\mathrm{kg} / \mathrm{m}^{2}\right)$. Patients were divided into six categories based on BMI (18.5-24.9, 25.0-26.9, 27.0-29.9, 30.0-34.9, 35.0-39.9 and $\left.\geq 40 \mathrm{~kg} / \mathrm{m}^{2}\right)$ (2).
The surgical procedure was performed with the patients under local anesthesia using a mini open incision technique. Local anesthesia was performed in each patient with an anesthetic agent containing lidocaine hydrochloride $(20 \mathrm{mg} / \mathrm{mL})$ and epinephrine $(0.0125 \mathrm{mg} / \mathrm{mL})$. Then, a $1-\mathrm{cm}$ vertical incision was made with a no. 15 scalpel, such that the distal end of the incision was located where the line was drawn from the middle of the third finger on the flexor side of the hand to the proximal and the line drawn from the medial side where the thumb ends intersect. After the automatic retractor was placed, blunt dissection was performed with a curved clamp. Dissection was continued after the transverse carpal ligament (TCL), which is the main part of the flexor retinaculum, was seen. After the distal border of the TCL was found, the median nerve was visualized by entering from beneath. Following the line with the median nerve, the entire TCL was cut toward the proximal over the median nerve. Finally, after homeostasis, the procedure was finalized by closing with a 3.0 Prolene Vicryl suture. Patients remained under observation for six hours after the procedure. They were then discharged with a broad-spectrum antibiotic prescribed, on the condition that they return for a follow-up visit 10 days later. Daily dressing change was recommended.

In the present study, the patients who underwent NCS six to nine months after the surgery were divided into two groups according to the postsurgical results and neurophysiological classification. Patients with mild CTS (partial recovery) or normal NCS (complete recovery) were defined as "surgical success" and those with moderate to severe CTS were defined as "surgical failure". This study was approved by the ethics committee of our institution (April 24, 2020, No. 2020/03-65), and the study was carried out in accordance with the Helsinki Declaration.

\section{Statistical Analysis}

Results are presented as mean \pm SD and percentage (\%). The normal distribution pattern of the data was confirmed using the Kolmogorov-Smirnov normality test. Patient age and BMI were compared using the Student's independent-samples $t$ test. Gender distribution and the frequency of DM were investigated using Fisher's exact test and chi-square test. The chi-square test was used to determine the relationship between the prevalence of DM in each BMI category and the BMl category. To investigate the independent predictive factors for surgical failure, a multivariate logistic regression model was used. Correlations between postoperative CTS severity and BMI and age were performed using the Spearman nonparametric correlation test because the postoperative CTS severity was ordinal. Results were interpreted according to the published principles of Cohen (weak positive correlation, $r=0.10-0.29$; moderate positive correlation, $r=0.30-0.49$; strong positive correlation, $r=0.50-1.00)$ (8). SPSS 23.0 (SPSS Inc., Chicago, IL) was used for all statistical analyses. A $p$ value less than 0.05 was considered statistically significant.

\section{RESULTS}

Forty-seven patients who underwent surgery for moderate 
CTS were included in the study. The group with surgical success consisted of 26 patients ( 7 men and 19 women; mean age, $47.3 \pm 10.9$ years), and the group with surgical failure consisted of 21 patients ( 4 men and 17 women; mean age, $54.3 \pm 12.6$ years). There was no difference in gender distribution between the groups ( $p=0.731)$; however, the mean age of patients with surgical success was significantly lower than those with surgical failure $(p=0.048)$.

The frequency of DM was $23.1 \%$ (6 of 26 patients) in patients with surgical success and $23.8 \%$ (5 of 21 patients) in patients with surgical failure, and this frequency did not differ between the groups $\left(x^{2}=0.003, p=0.953\right)$. In addition, the mean BMI of the patients with surgical success $\left(31.3 \pm 4.03 \mathrm{~kg} / \mathrm{m}^{2}\right)$ was significantly lower compared with the group with surgical failure $\left(36.75 \pm 5.5 \mathrm{~kg} / \mathrm{m}^{2} ; \mathrm{p}<0.001\right)$.

Although the prevalence of DM increased as the BMl level increased $(\mathrm{BMI}=18.5-24.9,0 \% ; \mathrm{BMI}=25.0-26.9$, no patients; $\mathrm{BMI}=27.0-29.9,16.67 \% ; \mathrm{BMI}=30.0-34.9,18.18 \%$; $\mathrm{BMI}=35.0-39.9,30.77 \%$; and $\mathrm{BMI} \geq 40,37.5 \%)$, this was not statistically significant $\left(x^{2}=2.668, p=0.615\right)$.

Table I presents the results of multivariate logistic regression analysis for surgical failure. Higher BMI $(p=0.003)$ and older age $(p=0.031)$ were found to be independent prognostic factors for surgical failure in patients with moderate CTS.

Moreover, a statistically significant and strong positive correlation was evident between postoperative CTS severity and BMl (rho=0.606, $\mathrm{p}<0.001$; Figure 1). However, no significant correlation was found between postoperative CTS severity and age $(\mathrm{rho}=0.226, \mathrm{p}=0.12)$.

\section{DISCUSSION}

In the current study, we found that age and BMI are independent prognostic factors for surgical failure in patients with moderate CTS. Specifically, patients with surgical success were younger and had a lower BMl as compared with patients with surgical failure. In addition, we found a statistically significant and strong positive correlation between postoperative CTS severity and BMI.

Both surgical and nonsurgical treatments are used to treat CTS $(11,16,25)$, and previous reports have indicated that surgical treatment is more effective than nonsurgical therapy $(11,20)$. However, even though surgery is effective, nearly $25 \%$ of patients do not benefit from this treatment (5). Patient- and surgeon-related causes of unsuccessful carpal tunnel surgery have been defined as follows: incomplete transection of the TCL, surgical site infections, postoperative palmar fascia necrosis, fibrous proliferation in the carpal tunnel, tenosynovitis of the flexor tendons, compression of the cutaneous branch of the median nerve, and, rarely, iatrogenic median nerve incisions $(6,12,15,22)$. However, to the best of our knowledge, the effect of BMI on the success of surgical treatment in CTS patients has not been thoroughly investigated in the literature. In the current study, we found that patients with low BMI benefit more from surgical treatment. In light of these important findings, we believe that delaying surgery for CTS
Table I: Multivariate Logistic Regression Analysis for Surgical Failure

\begin{tabular}{llc}
\hline & $\mathbf{p}$ & OR $(95 \% \mathrm{Cl})$ \\
\hline Age & $\mathbf{0 . 0 3 1}$ & $1.084(1.007-1.167)$ \\
Gender & 0.425 & $0.39(0.039-3.9)$ \\
BMI & $\mathbf{0 . 0 0 3}$ & $1.461(1.141-1.871)$ \\
DM & 0.09 & $6.26(0.74-52.4)$ \\
\hline
\end{tabular}

BMI: Body mass index, DM: Diabetes mellitus, OR: Odds ratio, Cl: Confidence interval.

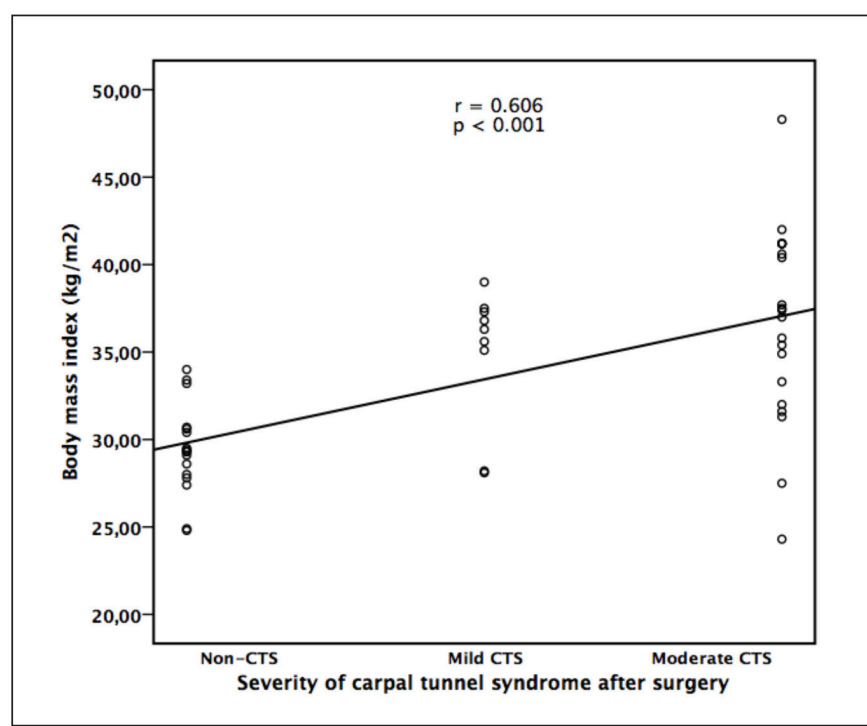

Figure 1: Graph of the correlation between the postsurgical severity of carpal tunnel syndrome and body mass index. Of note, the follow-up nerve conduction studies performed six months after the surgery showed that none of the patients developed severe carpal tunnel syndrome.

patients with a high BMI until after they lose weight would increase the success of surgical treatment.

Age is an important risk factor for CTS $(3,19)$. Moreover, a positive relationship was found between age and CTS severity (9). However, to the best of our knowledge, the correlation between age and outcomes of CTS surgery has not been investigated. In the present study, the mean age of the patients with surgical success was found to be significantly lower than the group with surgical failure. Age was an independent prognostic factor for surgical failure. This shows that age is an important risk factor for the development of CTS as well as for the surgical outcomes in CTS. However, age is an irreversible factor. Nevertheless, it is important for the surgeon or clinician to be aware of the fact that younger patients may benefit more and older patients may benefit less from CTS surgery. Likewise, the combination of surgical and nonsurgical methods may be more useful for the treatment of older patients with moderate to severe CTS. 
DM is known to be an important risk factor for CTS $(3,16,19)$. One meta-analysis found no significant difference between diabetic and nondiabetic patients in the recovery of all various outcomes, except for sensory conduction velocities after CTS surgery. In other words, in this analysis, a better result was observed in nondiabetic CTS patients only in sensory conduction velocity compared with diabetic patients (14). Similarly, other studies have found that after carpal tunnel release, diabetic patients have the same beneficial outcomes as nondiabetic patients $(23,26)$. Similar to these studies $(14,23,26)$, we found that DM had no effect on the outcomes of surgical treatment in CTS patients. However, some studies in the literature have indicated otherwise $(13,17)$. This is probably an issue that will continue to be discussed in the future. DM is known to be associated with BMI (2). However, we did not find a relationship between DM and BMI in our study. This result may be due to the small number of patients included in our study. We believe that further studies should be conducted with broader patient populations and longer follow-up periods to clarify this issue.

We believe that the findings of this study provide important contributions to the literature and also to the success of surgical treatment for CTS. However, there are some limitations to the current study. One limitation is that we did not include disease duration, occupational information of the patients, or information on other rare risk factors for CTS (such as connective tissue disorders). Another limitation is the fact that the study was retrospective and performed in only a single center.

\section{CONCLUSION}

In the present study, we found that age and BMI are important factors affecting the success of surgical treatment in CTS patients. Age is an unchangeable factor, but BMl can be changed. In CTS patients with high BMI who are considered for surgical treatment, success of the surgery can be increased by developing weight-loss strategies on an individual patient basis. We believe that the results obtained in the current study, which investigates the effect of age and BMI on surgical treatment results in CTS patients, will be confirmed in the future with further studies on this subject.

\section{ACKNOWLEDGMENTS}

The authors would like to thank Dr. A. Baygul and Assist. Prof. Dr. P. Gunes for their assistance with the statistical analyses.

\section{REFERENCES}

1. Atroshi I, Gummesson C, Johnsson R, Sprinchorn A: Symptoms, disability, and quality of life in patients with carpal tunnel syndrome. J Hand Surg Am 24(2):398-404, 1999

2. Bays HE, Chapman RH, Grandy S; SHIELD Investigators' Group: The relationship of body mass index to diabetes mellitus, hypertension and dyslipidaemia: Comparison of data from two national surveys. Int J Clin Pract 61(5):737-747, 2007
3. Becker J, Nora DB, Gomes I, Stringari FF, Seitensus R, Panosso JS, Ehlers JC: An evaluation of gender, obesity, age and diabetes mellitus as risk factors for carpal tunnel syndrome. Clin Neurophysiol 113(9):1429-1434, 2002

4. Bland JD: Carpal tunnel syndrome. BMJ 335(7615):343-346, 2007

5. Bland JD: Treatment of carpal tunnel syndrome. Muscle Nerve 36(2):167-171, 2007

6. Campagna R, Pessis E, Feydy A, Guerini H, Le Viet D, Corlobé P, Drapé JL: MRI assessment of recurrent carpal tunnel syndrome after open surgical release of the median nerve. AJR Am J Roentgenol 193(3):644-650, 2009

7. Chammas M, Boretto J, Burmann LM, Ramos RM, Neto FS, Silva JB: Carpal tunnel syndrome-Part II (treatment). Rev Bras Ortop 49(5):437-445, 2014

8. Cohen J: Statistical power analysis for the behavioral sciences. Abington, UK: Routledge, 1988

9. Gunes M, Buyukgol H: Correlation of neutrophil/lymphocyte and platelet/lymphocyte ratios with the severity of idiopathic carpal tunnel syndrome. Muscle Nerve 61:369-374, 2020

10. Huisstede BM, Fridén J, Coert H, Hoogvliet P; European HANDGUIDE Group: Carpal tunnel syndrome: Hand surgeons, hand therapist, physical medicine and rehabilitation physicians agree on a multidisciplinary treatment guidelineresults from the European HANDGUIDE Study. Arch Physical Med Rehabilit 95(12):2253-2263, 2014

11. Jarvik JG, Comstock BA, Kliot M, Turner JA, Chan L, Heagerty PJ, Hollingworth W, Kerrigan CL, Deyo RA: Surgery versus non-surgical therapy for carpal tunnel syndrome: $A$ randomised parallel-group trial. Lancet 374(9695):1074-1081, 2009

12. Karabay N, Toros T, Cetinkol E, Ada S: Correlations between ultrasonography findings and surgical findings in patients with refractory symptoms after primary surgical release for carpal tunnel syndrome. Acta Orthop Traumatol Turc 49(2):126-132, 2015

13. Kiylioglu N, Bicerol B, Ozkul A, Akyol A: Natural course and treatment efficacy: One year observation in diabetic and idiopathic carpal tunnel syndrome. J Clin Neurophysiol 26:446-453, 2009

14. Moradi A, Sadr A, Ebrahimzadeh MH, Hassankhani GG, Mehrad-Majd $\mathrm{H}$ : Does diabetes mellitus change the carpal tunnel release outcomes? Evidence from a systematic review and meta-analysis. J Hand Ther 33(3):394-401, 2020

15. Mosier BA, Hughes TB: Recurrent carpal tunnel syndrome. Hand Clin 29(3):427-434, 2013

16. Newington L, Harris EC, Walker-Bone K: Carpal tunnel syndrome and work. Best Pract Res Clin Rheumatol 29(3):440453, 2015

17. Ozer K, Malay S, Toker S, Chung KC: Minimal clinically important difference of carpal tunnel release in diabetic and nondiabetic patients. Plast Reconstr Surg 131(6):1279-1285, 2013

18. Padua L, Coraci D, Erra C, Pazzaglia C, Paolasso I, Loreti C, Caliandro P, Hobson-Webb LD: Carpal tunnel syndrome: Clinical features, diagnosis, and management. Lancet Neurol 15(12):1273-1284, 2016 
19. Pourmemari MH, Heliövaara M, Viikari-Juntura E, Shiri R: Carpal tunnel release: Lifetime prevalence, annual incidence, and risk factors. Muscle Nerve 58(4):497-502, 2018

20. Shi $Q$, MacDermid JC: Is surgical intervention more effective than non-surgical treatment for carpal tunnel syndrome? A systematic review. J Orthop Surg Res 6:17, 2011

21. Stevens JC: AAEM minimonograph \#26: The electrodiagnosis of carpal tunnel syndrome. American Association of Electrodiagnostic Medicine. Muscle Nerve 20(12):1477-1486, 1997

22. Steyers CM: Recurrent carpal tunnel syndrome. Hand Clin 18(2):339-345, 2002
23. Thomsen NO, Cederlund R, Rosén I, Björk J, Dahlin LB: Clinical outcomes of surgical release among diabetic patients with carpal tunnel syndrome: Prospective follow-up with matched controls. J Hand Surg Am 34(7):1177-1187, 2009

24. Werner RA, Andary M: Carpal tunnel syndrome: Pathophysiology and clinical neurophysiology. Clin Neurophysiol 113(9):1373-1381, 2002

25. Yunoki M, Kanda T, Suzuki K, Uneda A, Hirashita K, Yoshino $\mathrm{K}$ : Importance of recognizing carpal tunnel syndrome for neurosurgeons: A review. Neurol Med Chir (Tokyo) 57(4):172183, 2017

26. Zyluk A, Puchalski P: A comparison of outcomes of carpal tunnel release in diabetic and non-diabetic patients. J Hand Surg Eur Vol 38(5):485-488, 2013 